\title{
CONCERNING THE SPECIFIC RESPONSE OF GUINEA PIG'S RETICULOCYTES TO SUBSTANCES EFFECTIVE IN PERNICIOUS ANEMIA 1
}

\author{
By LOUIS S. GOODMAN, ARTHUR J. GEIGER AND THEODORE G. KLUMPP \\ (From the Departments of Pharmacology and Toxicology, and Internal Medicine, \\ Yale University School of Medicine, New Haven)
}

(Received for publication February 24, 1936)

Since the value of liver and stomach preparations in the treatment of pernicious anemia was first recognized, attempts have been made to evaluate the potency of the effective materials. Clinical trial, which is the only generally accepted method for testing these products $(1,18)$, is seriously handicapped by a growing scarcity of patients suitable for assay purposes. Bioassay methods employing laboratory animals have not proven successful. Forty-one such endeavors were listed in a recent review (9). Much interest, therefore, centers about the reports of Jacobson and coworkers $(7,8,9,10)$ that the normal guinea pig may serve as a valid indicator of the therapeutic potency of materials effective in pernicious anemia. Although the use of a normal animal appeared to violate a fundamental physiological principle of replacement therapy, it was deemed worth while to repeat Jacobson's experiments. Having an accumulation of experimental liver extracts awaiting potency determinations, we were eager to confirm the method and apply it to our needs. On the other hand, should the method prove unsatisfactory, an early recognition of this would be desirable. Reports on the potency of various chemically isolated fractions derived from liver and assayed by Jacobson's guinea pig method have already appeared in the literature $(3,20)$. From experiments based on this method, highly theoretical conclusions are also being drawn concerning the relation of congo red to the pathological physiology of pernicious anemia (13), and the reaction of normoblastic bone marrow to liver extract (11). Finally, the danger inherent in the use of an unreliable bioassay method by pharmaceutical firms is obvious.

1 Aided in part by a grant from the Committee on Scientific Research of the American Medical Association.

\section{METHODS AND PROCEDURE}

The method of Jacobson, as outlined in his latest publication (9), was followed precisely in the great majority of our experiments; all deviations will be specifically noted. In order that the following experiments may be clearly understood sufficient details of Jacobson's technique will be given.

Selection and care of animals. One hundred and eighteen normal adult guinea pigs weighing from 300 to 800 grams were used. About one-third of the animals were obtained from several local sources, and the remainder were received from reputable breeders in other cities. For convenience, we chose to work chiefly with male guinea pigs, as did Jacobson; only 20 were females. Wire cages with large-mesh screen bottoms housed from 4 to 8 tenants each. The diet consisted of oats, carrots and lettuce, as Jacobson recommended. In earlier experiments on 44 guinea pigs, cabbage was used in place of lettuce. A small number of animals, in an experiment to be described later, was placed on a diet producing black tongue in dogs.

Reticulocyte counting. In some preliminary experiments the "permanent preparation" method of Osgood and Wilhelm (17) was employed. This required about $0.05 \mathrm{cc}$. of blood obtained from a minute ear vessel; thin and well-stained preparations were easily made. In the great majority of experiments, however, the simpler "wet preparation" technique employed by Jacobson was followed.

Since we regarded a count of only 500 red blood cells, as employed by Jacobson, to be uncertain in accuracy for low reticulocyte ranges, our reticulocyte estimations were based on no less than 1000 , and frequently 5000 to 7000 cells. The counts were made by individuals experienced in the technique. Each preparation was treated as an "unknown," and it was a frequent practice for the observers to exchange slides for mutual checking. The reliability and uniformity of the results were thus insured.

Method of administering liver extracts. All solutions, with either water or normal saline as the diluent, were made up to a volume of $5 \mathrm{cc}$. and given intraperitoneally in a single dose. Lederle's Solution Liver Extract Parenteral, N.N.R., was used. Jacobson's basic dose was adopted, namely, the extract derived from $4.3 \mathrm{mgm}$. of fresh liver per $\mathrm{kgm}$. of guinea pig. In most experiments, 
however, 10, 100 and 240 times this amount was injected.

Control injections. In addition to potent liver extracts, two control materials were employed: sterile physiological saline solution and inactivated liver extract. Inactivation was accomplished by heating the liver solution to above $200^{\circ} \mathrm{C}$. for 30 minutes, and boiling the resulting charred material for 4 hours. A third control procedure consisted in daily reticulocyte counts on uninjected animals for extended periods.

Selection of reactive guinea pigs. The assay method of Jacobson is based on the use of so-called "reactive" guinea pigs; these animals are said to exhibit a specific reticulocyte response to liver extract. The criteria for their selection, and the fundamental characteristics of their reticulocyte behavior, as described by Jacobson, may be briefly summarized as follows:

1. If the reticulocyte values of an unselected group of newly acquired adult normal guinea pigs are followed for an initial test period of 8 days, it is stated that the large majority will consistently show levels below 1.2 per cent. Animals with reticulocyte percentages higher than 1.2, or those which do not fall to this level or lower and remain there for the last 4 days of this preliminary test period, are discarded as "unstable."

2. From 30 to 70 per cent of these stable animals are said to exhibit a reticulocyte response to potent liver extract. These guinea pigs comprise the group known as "reactive." This means that at some time within the 6 days following the injection, the reticulocytes should rise to 2.0 per cent or over for 2 consecutive days. Only animals that are reactive in this manner to the initial test dose of liver are retained for further use as assay subjects.

3. Guinea pigs once reactive are said to be permanently reactive to the injection of potent liver extracts, and by virtue of this remain suitable indefinitely for assay purposes.

Hematopoietic response to liver extracts. Inasmuch as stable and reactive guinea pigs, as defined above, are said to remain stable and to maintain indefinitely their specific reticulocytic responsiveness to liver with an approximately constant degree of sensitivity, Jacobson uses the reactive animals in the assay of unknown liver preparations. Furthermore, quantitative determination of potency is believed possible, since it appears that there is a threshold value for the response; and Jacobson places this minimally effective dose of potent liver at the amount of extract derived from $0.6 \mathrm{mgm}$. of fresh porcine liver per $\mathrm{kgm}$. of guinea pig. This amount is termed a guinea pig unit (G.P.U.). When a reactive guinea pig is given one G.P.U. or any multiple of this, the reticulocytes will rise within 6 days to 2.0 per cent or over for 2 consecutive days. It is stated that this response is all or none, and bears no relation to the amount of liver injected above the minimally effective dose; nor is there any other recognizable hematopoietic change of significance. In the assay of an unknown product, Jacobson accepts a positive response in 2 out of 3 injected test animals as evidence of the presence of the anti-pernicious anemia substance. By testing various known dilutions, the potency of the material can be quantitated. The reactive animals, after a suitable rest period, are used over and over again; one animal was used 18 times in 21 months by the author of the method.

Fundamental postulates of the method. It is obvious that in order for the guinea pig assay method to be valid, the two following fundamental criteria must be fulfilled unequivocally:

1. A "stable" animal must remain stable.

2. A "reactive" animal must show a reticulocyte response only to potent anti-anemia preparations, and not to indifferent substances; and it must remain reactive.

If either or both of these postulates cannot be substantiated, the method in our opinion is unsatisfactory.

\section{RESULTS}

To test the two basic tenets set forth above, 1977 reticulocyte counts were performed in 260 experiments on 118 guinea pigs.

Selection of "stable" guinea pigs. Our results show an extreme variability in reticulocyte levels of normal adult guinea pigs when observed for an eight-day period. This variability was observed not only in individual animals, but also in groups of guinea pigs. For example, in one group of 12 guinea pigs, 10 were stable on preliminary testing, but in another batch of 24 animals, only 1 was found stable.

When all our results on preliminary stability tests were analyzed nearly 48 per cent of all guinea pigs had reticulocyte levels which rendered them unsuitable in the sense described above. This figure is greatly in excess of that reported by Jacobson (9), who claimed that the large majority of all animals were stable on first trial. Regardless of this discrepancy, the question immediately arose whether the stable group, representing 52 per cent of our guinea pigs, would remain stable. We tried to answer this question in two ways. First, liver injection was withheld from a group of stable animals, and their reticulocytes were followed daily for from 8 to 20 days. Secondly, instead of discarding those found unstable on first trial, a large group was followed through second and third observation periods. In this manner it has been possible to learn much concerning the reticulocyte fluctuations occurring spontaneously in normal guinea pigs. 
In regard to the so-called stable group, 25 animals which met the requirements on first trial were allowed to rest for one or more weeks, and they were then re-examined for the stability of their reticulocyte levels. Thirteen were found to have more than 2.0 per cent reticulocytes for 2 consecutive days. This is at variance with the results of Jacobson (9), who reported that his stable group did not exceed 1.6 per cent reticulocytes when retested. If these animals had been injected with liver extract, these spontaneous reticulocyte rises might have led us to believe that over 50 per cent of the guinea pigs had responded positively. Typical charts of these experiments are shown in Figures 1 and 2, which illustrate a mild and an extreme instance, respectively, of spontaneous fluctuations of reticulocytes in previously stable animals.

It is obvious from these experiments that guinea pigs show spontaneous reticulocyte fluctuations of such a character that they cannot be depended upon to remain stable simply because they had had low reticulocyte counts during one trial period. Indeed, our results indicate that slightly

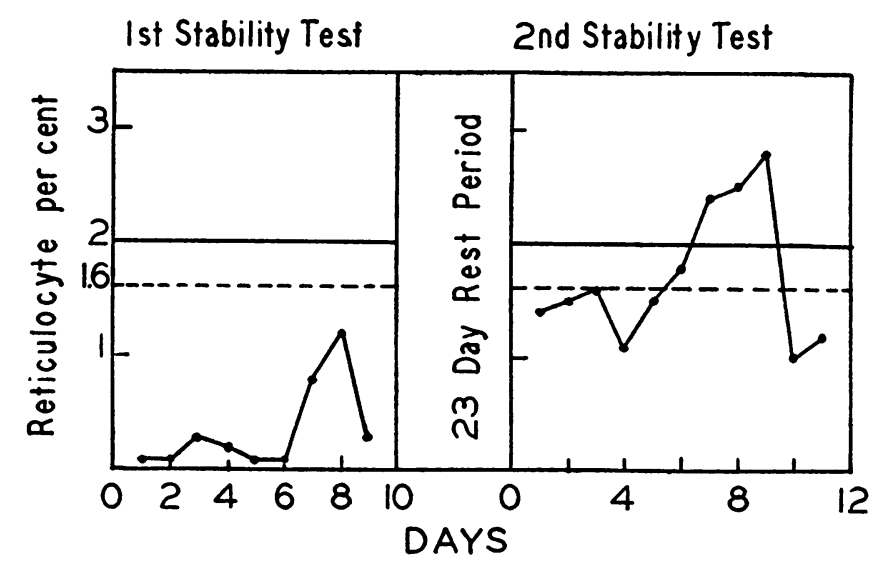

Fig. 1. Guinea Pig Number 17, do, 580 Grams. Mild Spontaneous Reticulocyte Fluctuation in a Previously Stable Animai.

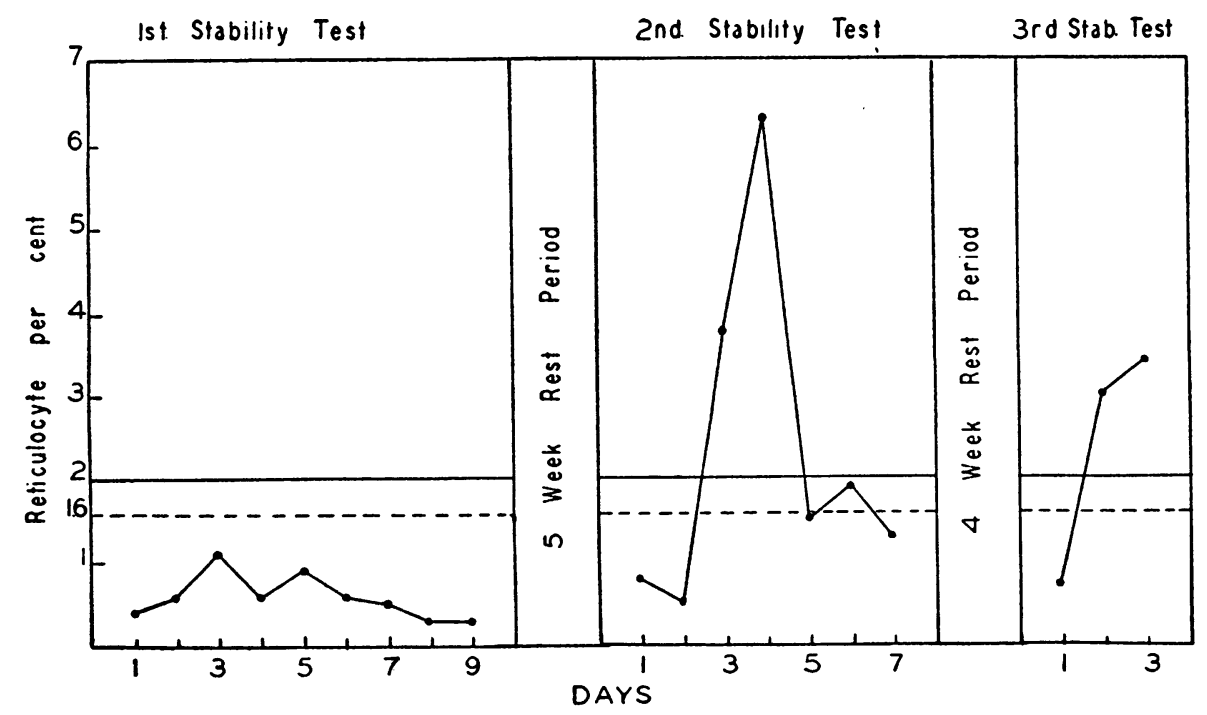

Fig. 2. Guinea Pig Number 25, ơ, 520 Grams. Extreme Spontaneous Reticulocyte Fluctuation in a Previously Stable Animal. 


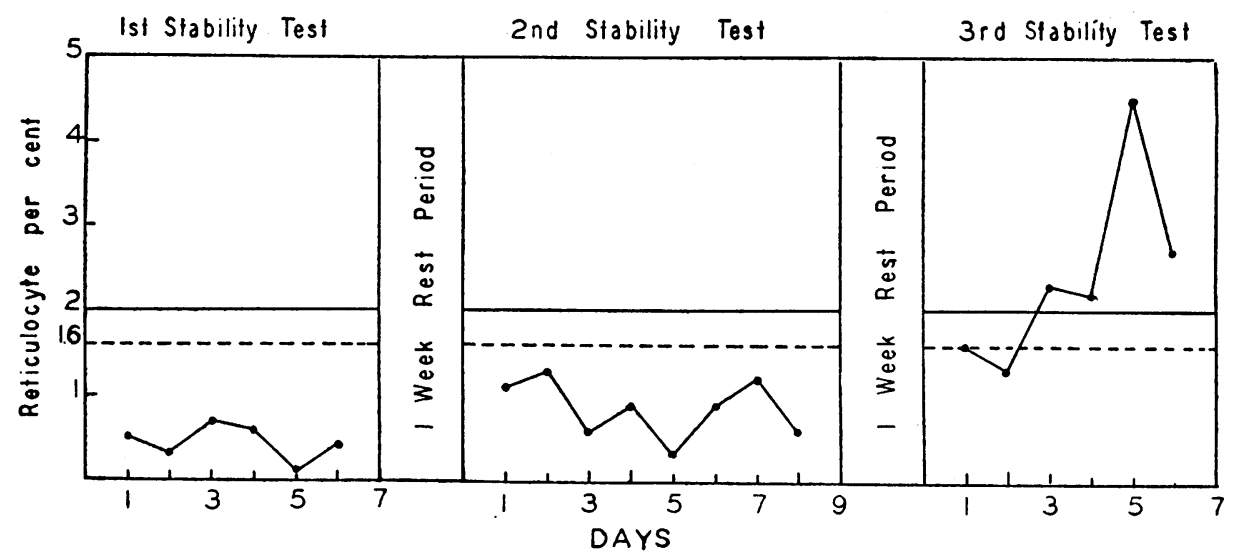

Fig. 3. Guinea Pig Number 50, $₫, 740$ Grams. Spontaneous Reticulocyte Fluctuation after Two Previous Stable Periods.

over 50 per cent of all guinea pigs stable during one observation period will be found unstable if re-examined at a later time. Furthermore, if one now takes those animals remaining stable through a second trial period and tests them a third time, again an appreciable number will be found unstable. For example, in one group of 11 guinea pigs stable during two previous observation periods, 5 were no longer stable when subjected to a third trial. In Figure 3, this occurrence is illustrated, and the experiment depicted is typical of 4 others.
From these experiments it is clear that in the guinea pig reticulocyte values fluctuate spontaneously. The reticulocyte behavior during any single period of observation, therefore, is not a valid basis upon which to label an animal stable or unstable. The particular result obtained depends entirely upon what part of the reticulocyte curve is being observed. If a stable guinea pig is subjected to repeated periods of observation, it will sooner or later be found unstable. This is well seen in Figure 4, which illustrates continuous daily reticulocyte counts taken on a stable animal over

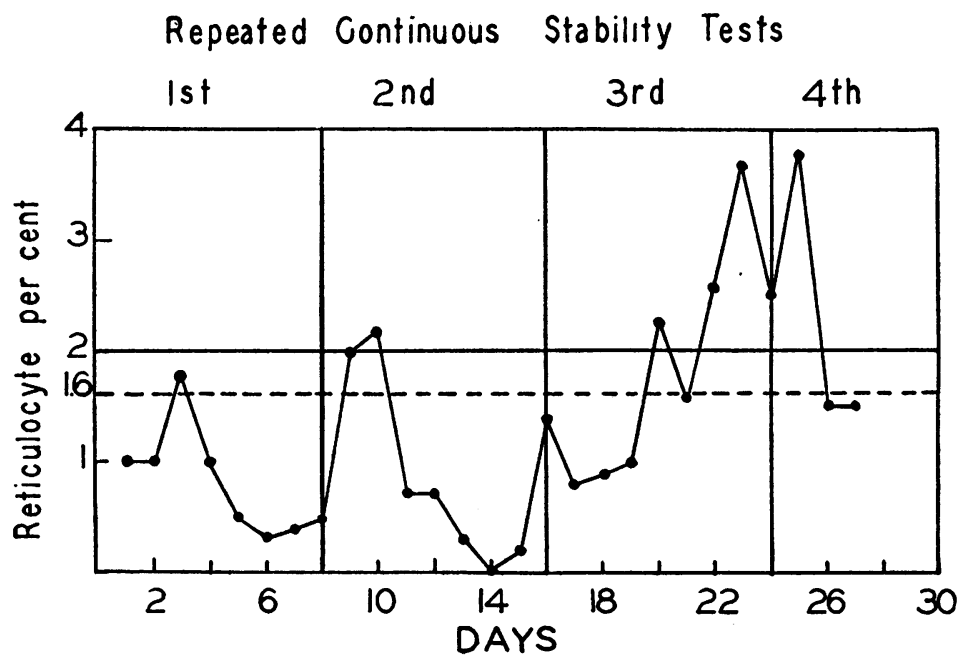

Fig. 4. Guinea Pig Number $14, \delta, 540$ Grams. Spontaneous Reticulocyte Fluctuations during Prolonged Observation of a Previously Stable Animal. 


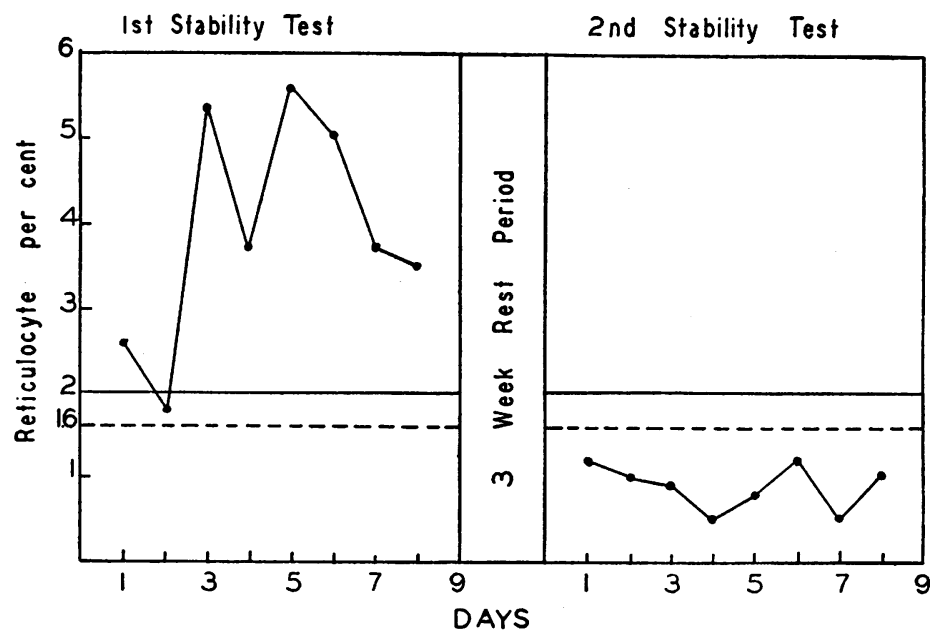

Fig 5. Guinea Pig Number 98, đ, 510 Grams. Stable Reticulocyte Period in a Previously Unstable Animal.

a period of 20 days, and is typical of 5 similar experiments.

Next, a study was made of the reticulocyte behavior of those animals which were unstable in the first observation period. Instead of being discarded, as Jacobson recommended, these animals were allowed to rest and were then reexamined. Of 29 such animals, 14 or slightly less than 50 per cent were found to be stable when given a second trial. Of the remaining 15 , which were again unstable during the second trial period, 10 were allowed to rest and then tested a third time. Of these, 6 animals were now found stable. In other words, only 4 animals of the original group of 24 that were followed remained unstable through three trials. It is probable that if these 4 animals were subsequently retested they too would ultimately have stable reticulocyte periods. The importance of these experiments lies in the possibility that if by chance all initially unstable guinea pigs had been observed a few weeks earlier or later, a large number would have been accepted as reliable test animals instead of having been discarded. Figure 5 shows an experiment on an unstable guinea pig, which, when tested three weeks later, was found stable; this experiment is typical of 13 similar ones. Figure 6 illustrates that an animal, unstable during two separate trial periods, was found stable on the third testing; there were 5 experiments with similar results.

From the above evidence, it may now be said that not only is it impossible to rely on the reticulocyte constancy of a so-called stable animal, but

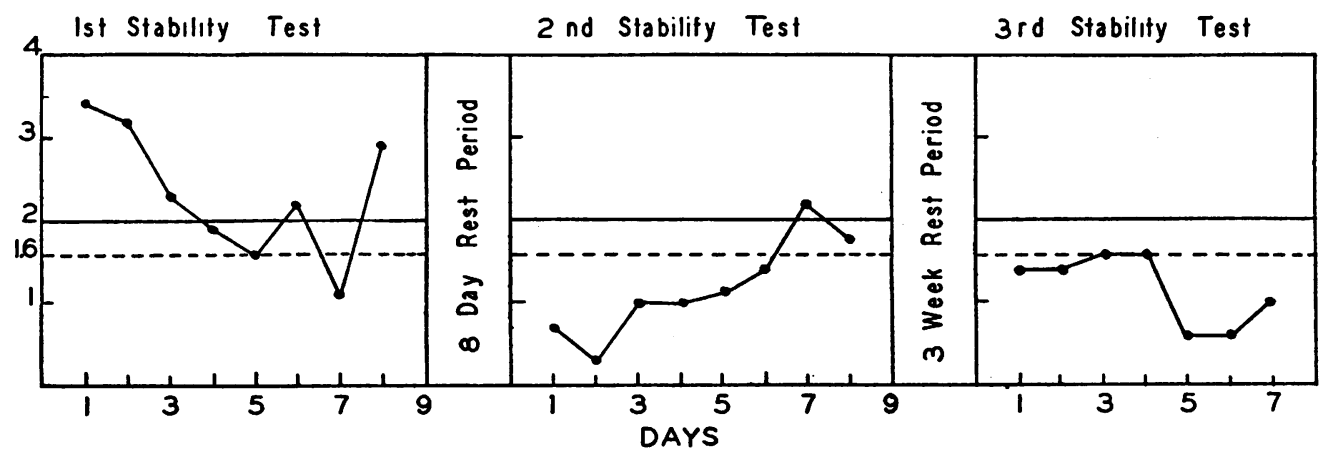

Fig. 6. Guinea Pig Number 105, ơ, 480 Grams. Stable Reticulocyte Period after Two Previous Unstable Periods. 
it is also impossible to discard a particular guinea pig as unstable. The results, in our opinion, definitely indicate that if one is guided by the criteria of the method, all guinea pigs are unsuitable because of their normal, spontaneous and unpredictable reticulocyte fluctuations. It has not been possible, therefore, to substantiate the first postulate of the method.

\section{The specificity of the reticulocyte response to liver injections}

I. Response to injection of liver. Despite the marked and unpredictable fluctuations of reticulocytes occurring in normal guinea pigs, we deemed it advisable to investigate the method further to discover whether there might be some characteristic response to potent liver easily distinguishable from any spontaneous change in reticulocyte level.

After a short rest period, potent liver extract was administered to 44 guinea pigs meeting the qualifications for stability. The experiments were divided roughly into four groups given respectively Jacobson's routine dose and also 10, 100, and 240 times this dose. Positive responses were obtained in 15 experiments, or 34 per cent. No difference was noted in regard to the amount of liver injected. The group receiving the smallest dose showed the same variability of reticulocyte behavior as the group receiving the largest dose. In Figure 7 is illustrated a positive and negative experiment in the same animal which on two occasions, separated by a suitable rest period, received 240 times the routine dose of liver extract. A positive reticulocyte response appeared to follow the first injection; there was no apparent effect from the second injection. A similar result was obtained in 2 other experiments.

These experiments indicate that a guinea pig which has responded positively cannot be relied upon thereafter always to respond positively to liver. Jacobson's contention, therefore, that " the reactive state is maintained indefinitely" was not confirmed.

II. Response to injections of control substances. Observations made on normal uninjected guinea pigs constitute important control data. Additional control experiments were performed by injecting isotonic solutions of sodium chloride and totally inactivated liver extract. The latter was given in doses corresponding to those used for the potent extracts. The method of inactivation has been described.

Injections of salt solution were made in 27 stable guinea pigs. Many of these animals had undergone prior injections of liver or repeated stability observations; the remainder received the saline injection directly following the first stability test. Ten of these 27 experiments resulted positively, and thus 37 per cent of the nonspecific saline injections produced the type of response reserved by the Jacobson method exclusively for potent liver materials.

Injections of inactivated liver were made in 16 stable guinea pigs, and 10 of these, or 63 per cent,

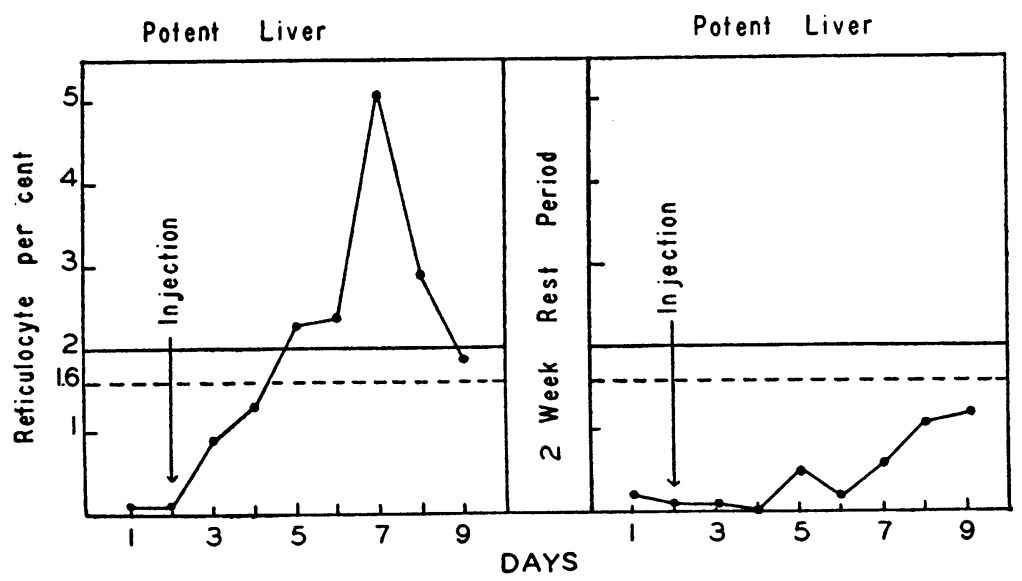

Fig. 7. Guinea Pig Number 18, ${ }^{*}, 445$ Grams. Negative Response to Liver Injection in a Previously Reactive Animal. 
were positive. In other words, the majority of the animals reacted positively to impotent liver.

From these control results, it appears that here again one is observing only normal spontaneous fluctuations of reticulocytes, and that the injections of liver extract or saline are irrelevant and merely coincidental.

In Figure 8 is shown an example of a positive response to the injection of saline solution.

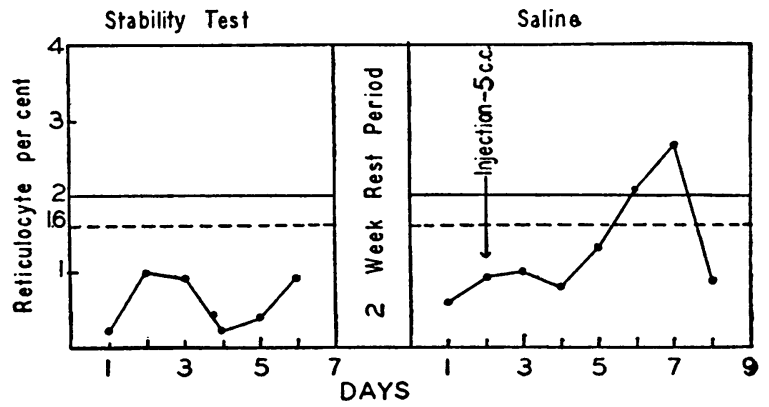

Fig. 8. Guinea Pig Number 54, $\delta, 535$ Grams. Positive Response to Saline Injection.

Figure 9 illustrates a positive response to the injection of inactivated liver extract solution.

In Figure 10 is depicted a control experiment in a stable animal which had reacted positively to the injection of liver. Then, to test the specificity of the liver response, saline and inactivated liver injections were given. Positive reticulocyte responses followed each of the control injections. This experiment is typical of 5 others.

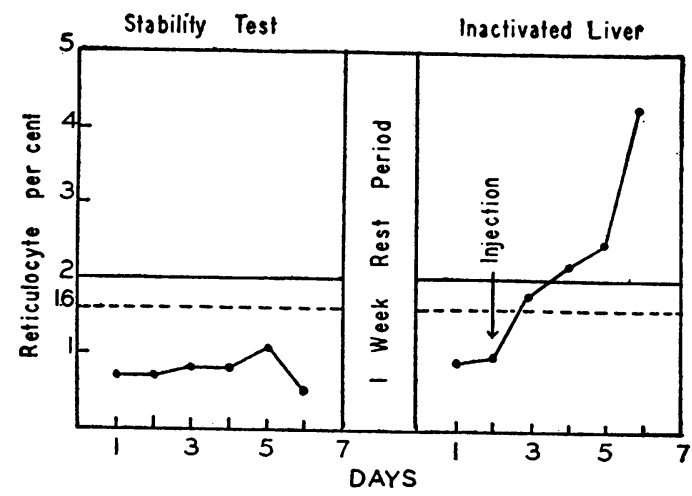

Fig. 9. Guinea Pig Number 48, $\delta, 625$ Grams. Positive Response to Inactivated Liver Injection.

A comparison was made of the reticulocyte curves of all positive responses including those occurring spontaneously and after active liver, inactivated liver, and saline injections. The analysis included the character of the curves in regard to contour, the average height of reticulocyte peaks, the total reticulocyte response, the duration of the response, and the day on which the peak was reached. No features were found which would distinguish the potent liver responses from the non-specific ones. The results are listed in Table I.

From the evidence presented in this section, therefore, it is clear that the second postulate concerning the specificity and permanency of the reticulocyte response to liver extract could not be substantiated.

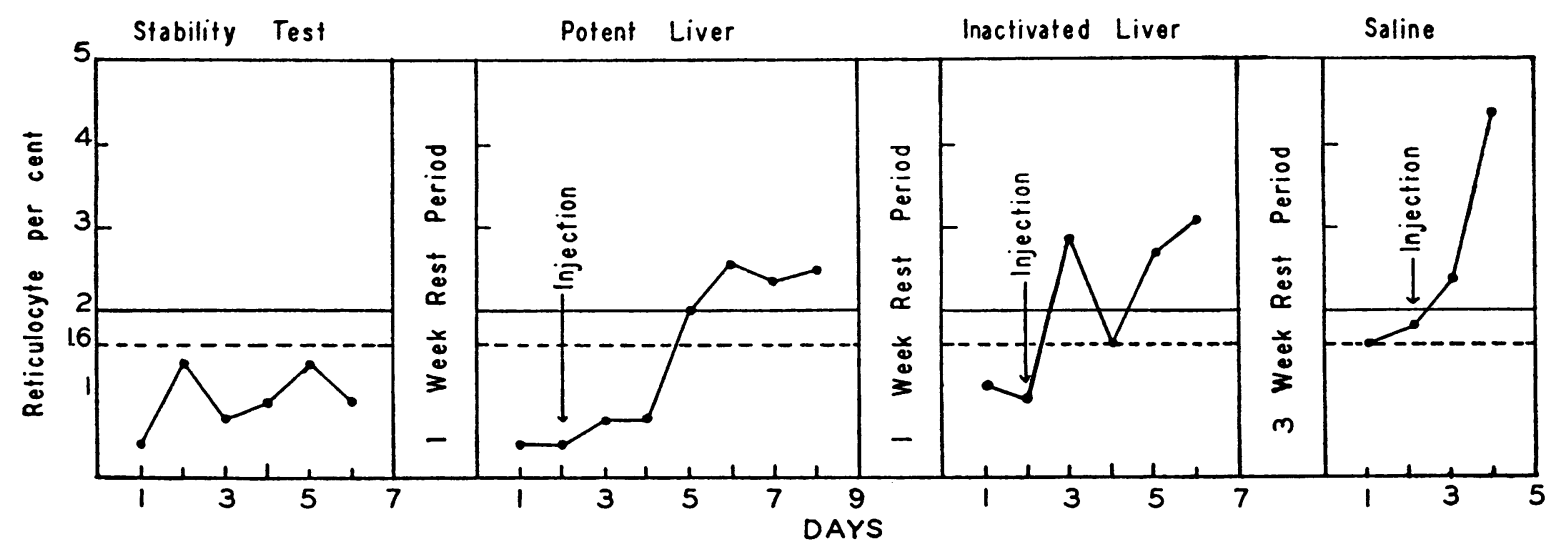

Fig. 10. Guinea Pig Number 46, đૈ, 580 Grams. Positive Responses to Inactivated Liver and Saline in a Previously Stable and Reactive Animal. 
TABLE I *

Analysis of positive reticulocyte responses in stable guinea pigs

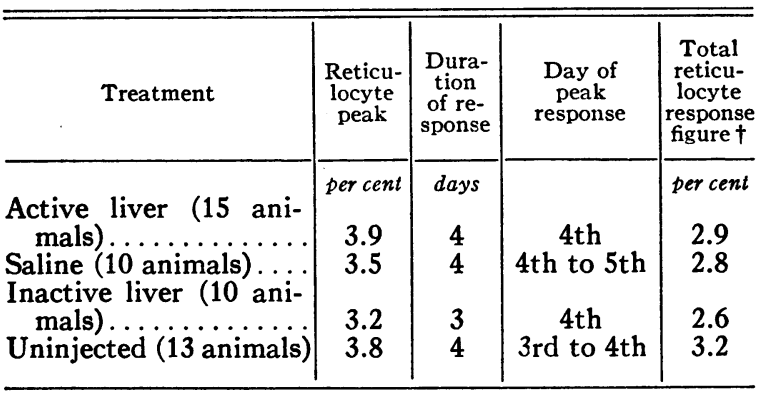

* All figures represent the average of the values for all experiments in each group.

$\dagger$ This figure is obtained by averaging those reticulocyte values over 1.8 per cent during the period of response.

The method, we believe, has established by definition an arbitrary division between stable and unstable guinea pigs, and positive and negative responses. It is our experience that this dividing line of 1.2 per cent reticulocytes, which differentiates between stable and unstable animals, is drawn near the mean in the range of reticulocyte fluctuations of normal guinea pigs. For example, if our interpretation is correct concerning the spontaneous character of these reticulocyte values, it would be of interest to know the range of distribution of the reticulocyte counts obtained in all our experiments. This is shown in Figure 11, in which curve $A$ represents the distribution of 1977 reticulocyte counts done on 118 guinea pigs regardless of the various procedures followed in particular animals. It is interesting that 1029 counts, or 52 per cent of the total, are 1.2 per cent or below. This correlates well with the previous finding that approximately one-half of our guinea pigs were stable.

In Figure 11, curve $B$ represents the distribution of the reticulocyte counts of all the potent liver experiments; curve $C$, similarly, represents the distribution of the reticulocyte counts of all the control experiments. There is no significant difference in the character of these reticulocyte distributions.

It is merely chance that in any fairly large group of animals, regardless of various harmless procedures, a certain number will show reticulocyte values over 2 per cent for 2 consecutive days. The definition of a positive response is, in our opinion, an arbitrary one.

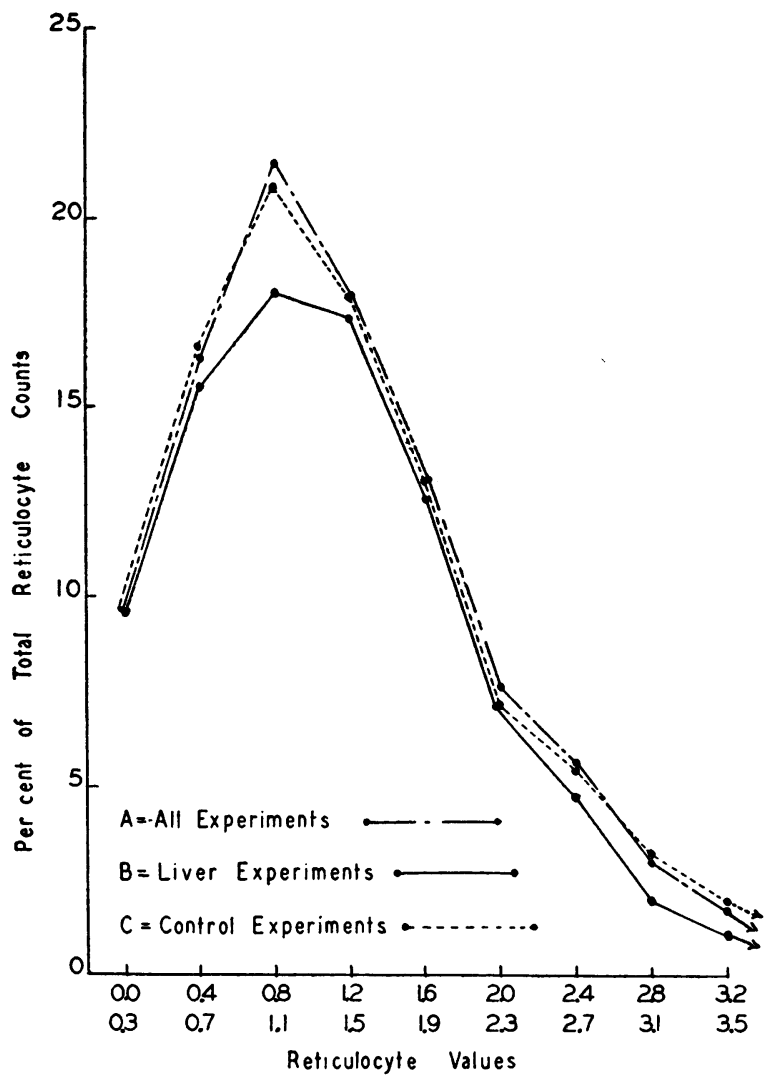

Fig. 11. Distribution of 1977 Reticulocyte Counts Representing All Experiments (Curve $A$ ), and ComPARISON WITH 439 Counts IN Liver ExpERIMENTS (Curve $B$ ), and 1538 Counts in Control Experiments (Curve $C$ ).

In Table II is presented a statistical summary of all the experiments performed on stable guinea pigs.

TABLE II

Statistical summary of results of experiments performed on stable guinea pigs

\begin{tabular}{|c|c|c|c|c|c|}
\hline \multirow{2}{*}{ Treatment } & \multirow{2}{*}{$\begin{array}{l}\text { Number } \\
\text { of } \\
\text { experi- } \\
\text { ments }\end{array}$} & \multicolumn{2}{|c|}{$\begin{array}{c}\text { Positive } \\
\text { responses }\end{array}$} & \multicolumn{2}{|c|}{$\begin{array}{l}\text { Negative } \\
\text { responses }\end{array}$} \\
\hline & & $\underset{\text { ber }}{\text { Num- }}$ & $\begin{array}{l}\text { Per } \\
\text { cent }\end{array}$ & $\underset{\text { ber }}{\text { Num- }}$ & $\begin{array}{l}\text { Per } \\
\text { cent }\end{array}$ \\
\hline 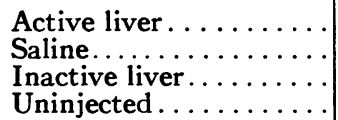 & $\begin{array}{l}44 \\
27 \\
16 \\
25\end{array}$ & $\begin{array}{l}15 \\
10 \\
10 \\
13\end{array}$ & $\begin{array}{l}34 \\
37 \\
63 \\
52\end{array}$ & $\begin{array}{r}29 \\
17 \\
6 \\
12\end{array}$ & $\begin{array}{l}66 \\
63 \\
37 \\
48\end{array}$ \\
\hline
\end{tabular}

Results in regard to sex, source and diet. No differences were noted in groups of guinea pigs acquired from different sources. The data presented by males and females were identical. The 
results in the preliminary experiments with 44 guinea pigs given cabbage as the green vegetable were the same as the results with the 74 guinea pigs fed lettuce; nor were there any changes when the cabbage-fed animals were later placed on lettuce.

Hematological observations. In 23 animals the main features of the blood picture were studied. Red cell counts were done in duplicate, using certified blood pipettes and counting chambers. Hemoglobin readings were made on the HadenHauser hemoglobinometer calibrated by the oxygen capacity determination. Packed red cell volumes were measured with Van Allen hematocrits, employing 2 per cent potassium oxalate solution. The mean and extreme findings are presented in Table III.

TABLE III

Hematological data on 23 normal guinea pigs

\begin{tabular}{|c|c|c|c|}
\hline & $\begin{array}{l}\text { Red blood } \\
\text { cell count }\end{array}$ & Hemoglobin & Hematocrit \\
\hline $\begin{array}{l}\text { Average } . \ldots \ldots \ldots \ldots \\
\text { Range } . . \ldots \ldots \ldots \ldots\end{array}$ & $\begin{array}{c}\text { millions } \\
6.10 \\
7.04-4.73\end{array}$ & $\begin{array}{c}\underset{\text { grams }}{\text { per cent }} \\
14.7 \\
16.0-13.0\end{array}$ & $\begin{array}{c}\text { per cent of } \\
\text { packed cells } \\
42.0 \\
47.0-37.0\end{array}$ \\
\hline
\end{tabular}

These figures compare favorably with the values presented by Jacobson, and suggest that our animals were not hematopoietically different from his guinea pigs. We found no correlation between blood pictures, reticulocyte variations, and treatment. The level of the circulating reticulocytes is usually regarded as a sensitive index of bone marrow activity. The rise and fall of these cells in our experiments were not correlated with any increased or decreased demand detectable in the blood picture. This again suggests that the variable reticulocyte values in these animals represent only the usual activity of the normal guinea pig's marrow. It is not remarkable, then, that in the absence of a specific hematopoietic deficiency, no characteristic response follows the injection of liver extract.

Results in regard to reticulocyte counting method. When a comparison was made of the data obtained by the "permanent preparation" method of Osgood and Wilhelm (17), which was used in about 20 per cent of our experiments, and the "fresh preparation" method used in the remainder, no significant differences were noted.
The small daily loss of blood incidental to the reticulocyte estimations had no effect on the reticulocyte values or blood count. This was determined in 6 animals that were bled daily far in excess of the amount of blood required for the reticulocyte counts.

In our opinion a reticulocyte percentage based on a count of only 500 red blood cells is unreliable in these low reticulocyte ranges. Unusual care was exercised to insure perfection in the preparation of the blood films; nevertheless, the values obtained for duplicate reticulocyte counts on blocks of 500 cells each were often significantly different. The necessity for counting at least two blocks of not less than 500 cells each is seen in Figure 12 which illustrates a few of the more striking instances in which, by counting only 500 cells, one might conclude that the animal was stable or non-reactive, and then by counting a different block of 500 cells the animal would be judged unstable or reactive.

We do not feel that the presentation of these data labels our counting technique any the less accurate, but rather that it indicates a source of difficulty and possible error in a relatively simple hematological procedure even when done carefully by experienced workers. It is our understanding that the accepted standard for reticulocyte counting in clinical work is a minimum of 1000 red blood cells for reticulocyte levels under 5 per cent. Since Jacobson's method necessitates the differentiation between 1.5 and 2.0 per cent of reticulocytes, permitting an error of less than 3 reticulocytes per 500 red cells, it would seem even more necessary to examine at least 1000 cells.

Reticulocyte behavior of guinea pigs on the Goldberger diet producing black tongue in dogs. Inasmuch as we were unable to obtain truly stable guinea pigs, it was thought desirable to attempt to stabilize the circulating reticulocytes at a low level and to render the bone marrow specifically susceptible to liver extract. We sought to accomplish this by feeding a deficient diet. The results of some of these experiments (2) are reported here because they shed additional light on the bone marrow activity of this species. The many lines of evidence which suggested the use of the diet producing pellagra can be indicated only briefly here. In Goldberger's hands the diet 


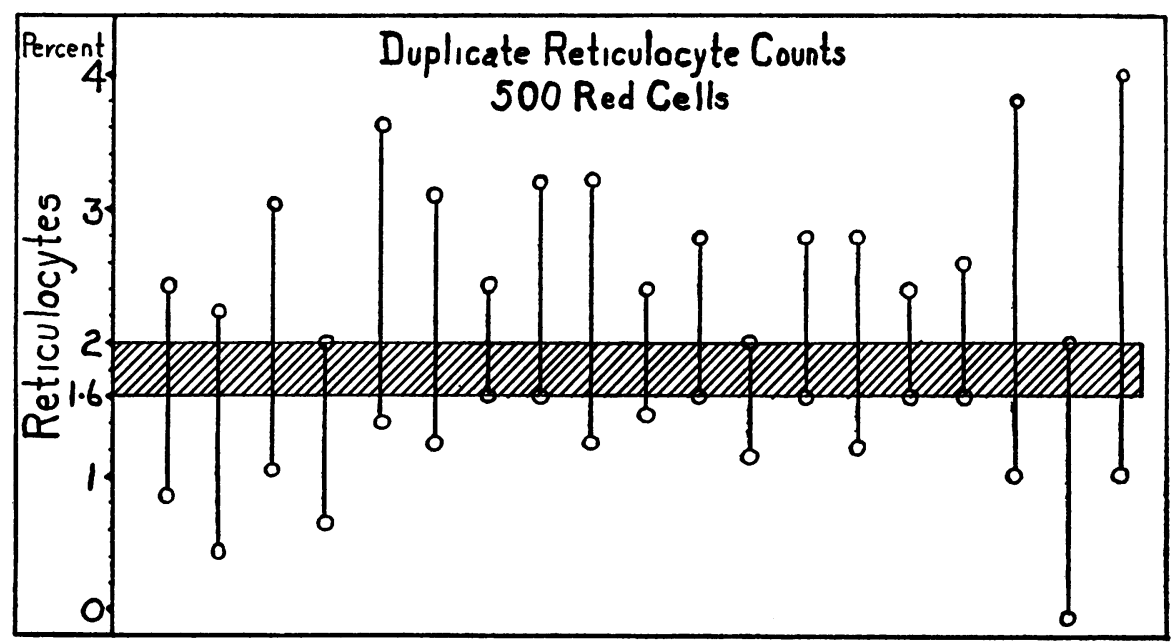

Fig. 12. 19 Representative Instances of Significant Differences in Duplicate Reticulocyte Counts, of 500 Cells Each, Made on the Same Preparation.

Note that in each instance the duplicate counts fall on opposite sides of the shaded "crucial zone" which defines reactivity and the upper limit of stability.

was productive of pellagra in humans (5). Rhoads and Miller (19) reported that this diet caused anemia and black tongue in dogs, while in swine it produced a syndrome strikingly similar to human pernicious anemia (15). The diet has been thought to be deficient in vitamin $B_{2}$, and the relationship between the anti-pernicious anemia principle and vitamin $B_{2}$ has received considerable attention. Finally, Miller and Rhoads found that the anti-anemia substances, when fed orally, possessed a life-saving factor for guinea pigs maintained on this diet (14).

Guinea pigs were fed the diet producing pellagra (4, Table 6) plus orange juice, but omitting sucrose, and their blood pictures were followed. In the majority of these animals the reticulocytes decreased within two weeks to extremely low levels. In many instances no reticulocytes were seen even on counting as many as 5000 to 10,000 red blood cells, and counts of 0.02 per cent were not rare. In 4 animals showing such extremely low counts for from several days to a week, single intraperitoneal injections of $0.25 \mathrm{cc}$. of Lederle's concentrated liver extract solution were given. The reticulocytes rose rapidly in 3 guinea pigs, more slowly in the fourth one, but in all instances there occurred what appeared to be a definite and marked response with an increase of reticulocytes to from 2.0 to 2.5 per cent. As a control, there- fore, another group of 4 animals on the same diet was observed daily, and it was noted that there occurred spontaneous increases in reticulated cells much greater than those observed after liver extract. In 2 animals that lived on this diet for two months, several periods of alternating extremely low and very high reticulocyte levels were seen, and these phasic variations were related neither to any observable change in the well-being

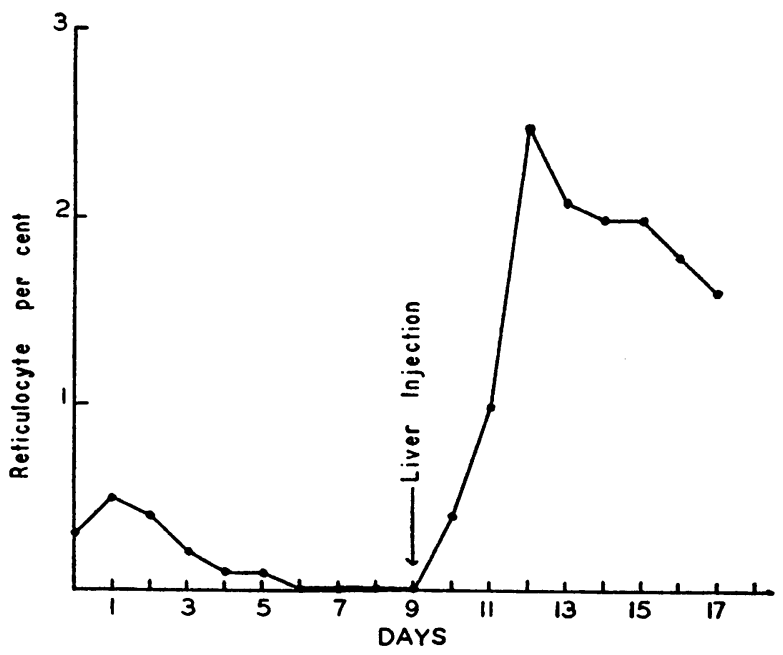

Fig. 13. Guinea Pig Number 110, ð, 320 Grams. Goldberger Diet. Apparent Positive Response to LIVER INJECTION. 
of the guinea pigs nor to the loss of weight which occurs on this regimen. Here again, then, one finds marked fluctuations in animals which for short periods of observation appear to have reduced bone marrow activity and low and stable reticulocyte values induced by a deficient diet. Figure 13 illustrates an apparent effect of liver injection given during a period of reduced bone marrow activity, and Figure 14 presents a comparable experiment in which liver injection was withheld but the reticulocytes counted daily for over two months.

From these experiments it seems that this diet altered bone marrow activity in such a manner that the swings in peripheral reticulocyte levels seen in normal guinea pigs were markedly accentuated. The reticulocyte behavior on the deficient diet differed not in kind but only in degree from that of normal animals. Thus, what appeared to be a promising method for rendering the guinea pig a suitable assay animal has failed in our hands.

It should be added that the daily oral administration of large amounts of Lilly's Liver Extract, N.N.R., to these guinea pigs had no characteristic effect on the reticulocytes. The response obtained by Jacobson (9) from the oral admin- istration of anti-anenia substances to normal guinea pigs finds no support in these experiments.

\section{DISCUSSION}

The failure of our experiments to corroborate Jacobson's assay method is clear from the results presented. This discrepancy cannot reasonably be ascribed to an inherent hematological difference between his animals and ours. We used normal adult guinea pigs deliberately obtained from several sources. The animals remained healthy, and the blood pictures in a representative group were entirely normal throughout the experiments.

Jacobson (9) suggested that the megaloblastic bone marrow which he observed in his reactive animals bore a close causal relation to their suitability for assay purposes, and stated that "the large number of megaloblasts in proportion to the number of normoblasts, simulate the classical picture of the bone marrow findings in pernicious anemia." $\mathrm{He}$ found, however, the same type of marrow in the non-reactive guinea pigs, and from the remainder of the hematological data it is probable that his finding is the normal condition for this species. A megaloblastic marrow oc-

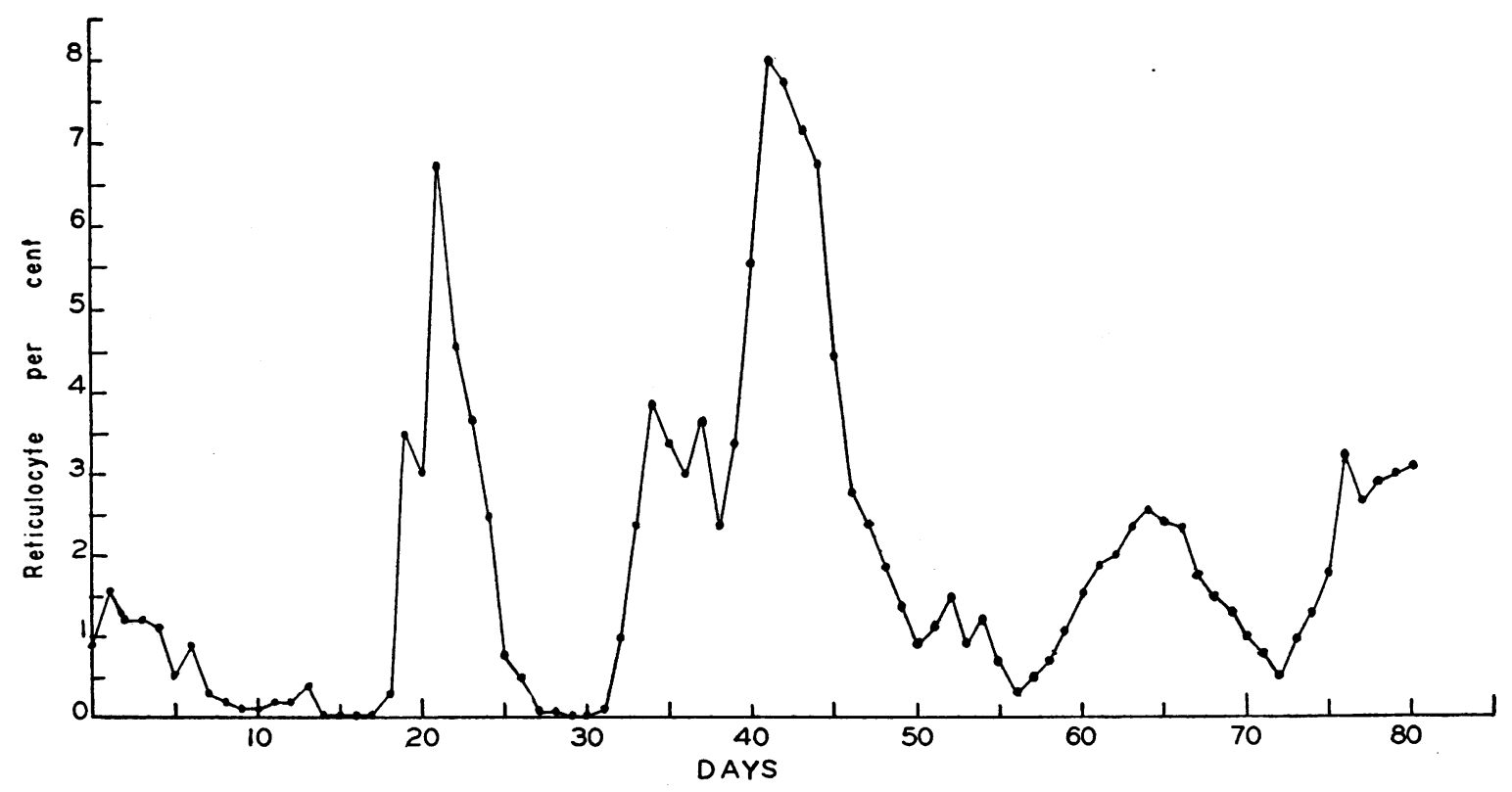

Fig. 14. Guinea Pig Number 114, o, 535 Grams. Goldberger Diet. Marked Spontaneous Swings in Reticulocyte LeVELS. 
curring normally in a non-anemic guinea pig is obviously entirely different in significance from the abnormal megaloblastic hyperplasia seen in pernicious anemia patients in relapse. It may be recalled here that once previously the impression that a megaloblastic marrow in a normal animal rendered that species suitable for liver assay purposes resulted in much futile work on pigeons. ${ }^{2}$

It would appear at present that there is no physiological basis for the development of a specific reticulocytosis to liver in animals with normal blood, and in our experience the guinea pig is no different in this respect from the other species of normal laboratory animals which have failed to yield a bioassay method for anti-pernicious anemia substances.

A search of the literature revealed only two reports apparently confirming the usefulness of the normal guinea pig as a test animal. The first study, by Landsberg and Thompson (12), was based on only 6 animals, and no controls were mentioned. In 4 of their 6 animals the pre-injection reticulocyte levels would render them unsuitable for the method here under consideration. The report of Miller and Rhoads (16) deserves special mention because these workers implied confirmation of Jacobson's results and stated that "increases in the number of circulating reticulocytes which are similar in degree, duration, and time of occurrence to those obtained by Jacobson may be induced by potent, anti-anemic substances which are orally as well as parenterally administered." A careful analysis of their data, however, reveals that their results differ from Jacobson's in important respects. In the first place, the height of reticulocytosis obtained by Miller and Rhoads was definitely greater than Jacobson's. The latter's positive responses were under 3.4 per cent in 90 per cent of 193 experiments, whereas, in Tables II and III presented by Miller and Rhoads, at least 5 responses out of the 11 recorded were over 3.4 per cent, and in one

\footnotetext{
2 It is of interest to note that very recently Jones (11) investigated the bone marrow of the guinea pig, pigeon, and rat. He found that the red cells of these marrows "belong to the definitive or normoblastic series, and they are identical with those of the marrow of the normal human adult, which does not possess megaloblasts." There was "-no morphologic evidence for a megaloblastic bone marrow in the guinea pig," and no similarity to the megaloblastic marrow of pernicious anemia.
}

experiment all 3 animals reached 5 per cent or over. Since the method deals essentially with low reticulocyte values, such a difference in one of its fundamental phenomena appears significant. More important, however, is the fact that Miller and Rhoads' reticulocyte responses did not occur until the seventh to thirteenth day after the initiation of treatment, while Jacobson's criterion of a positive response demands that it occur within six days. The importance of this difference is well illustrated in Jacobson's Table VII (9) which shows that animal 256 did not respond within six days to a presumably impotent material given orally. This animal was then immediately given a second mixture, and this time a reticulocyte response did occur within the required period of six days. On the basis of these time relationships, this so-called positive response, coming as it did on the twelfth day after the original impotent material, was regarded by Jacobson as evidence of potency of the second and impotency of the first preparation. Miller and Rhoads, on the other hand, might readily have regarded the first preparation as potent, because they accepted such late reticulocyte responses as positive. Indeed, inasmuch as all the positive responses obtained by Miller and Rhoads occurred after the first week, these same positive responses would, if judged by Jacobson's criteria, be called negative responses.

These discrepancies in the degree and time of appearance of the reticulocytosis cannot be ascribed to differences in dosage. Jacobson (9, Table V) noted no influence on the magnitude of the reticulocyte rise after the injection of the minimal effective dose as compared with 6000 times this quantity. Furthermore, he noted a tendency for the responses to occur earlier, if anything, within the six-day observation period when larger amounts of liver were given. Our own interpretation of these data is that here again one is encountering nothing more than the usual non-specific and spontaneous reticulocyte fluctuations of normal guinea pigs. ${ }^{3}$

\footnotetext{
${ }^{3}$ In a recent personal communication C. P. Rhoads has written, "Further experimentation has advanced incontrovertible evidence that guinea pig reticulocytes may increase to a level of over 2 per cent from a variety of causes unassociated with the administration of substances effective in the treatment of pernicious anemia. Experiments are under way in an attempt to elucidate
} 
We have carefully observed the four precautions listed by Jacobson in his discussion (9) in regard to the diet and environment of the pigs, the high degree of accuracy in reticulocyte counting, the selection only of animals stable during a preliminary control period for liver injection, and the use of liver extract of a high degree of therapeutic potency. Despite meeting these and the other criteria of the method, on the basis of our experience we are forced to conclude that the normal guinea pig exhibits, in respect to his reticulocytes, such a capricious behavior that the use of this species for bioassay of liver extract is at present unsuitable and hazardous.

The prediction is ventured that a satisfactory laboratory assay animal will not be obtained until it is possible to produce experimentally a clinical picture closely simulating the pernicious anemia of man. Whether the anemia of swine produced by Miller and Rhoads (15), or the anemia associated with liver cirrhosis obtained in rats by Higgins and Stasney (6), will provide the longsought laboratory test animal remains a question for further experimentation.

\section{CONCLUSION}

The normal adult guinea pig shows considerable and unpredictable spontaneous fluctuations in reticulocyte levels. These variations are of such a nature as to render this normal animal unsuitable for assaying the potency of materials effective in pernicious anemia.

The authors are grateful to Mrs. Helen Goodman for her invaluable technical assistance.

\section{BIBLIOGRAPHY}

1. Dameshek, W., and Castle, W. B., Assay of commercial extracts of liver for parenteral use in pernicious anemia; method of successive reticulocyte responses in the same patient. J. A. M. A., 1934, 103, 802.

2. Doeller, C., Goodman, L. S., and Geiger, A. J., (Experiments to be published).

3. Fiske, C. H., Subbarow, Y., and Jacobson, B. M., The multiple nature of the pernicious anemia principle in liver. J. Clin. Invest. (Proc.), 1935, 14, 709.

4. Goldberger, J., Wheeler, G. A., Lillie, R. D., and Rogers, L. M., A further study of butter, fresh

the cause of spontaneous variations in numbers of reticulocytes; such spontaneous variations demand that the greatest care be used in employing the guinea pig as a test for anti-anemic substances." beef, and yeast as pellagra preventives, with consideration of the relation of factor P-P of pellagra (and black tongue of dogs) to vitamin B. U. S. Pub. Health Rep., 1926, 41, 297.

5. Goldberger, J., and Wheeler, G. A., The experimental production of pellagra in human subjects by means of diet. Nat. Inst. Health Bull., 1920, No. 120, 7.

6. Higgins, G. M., and Stasney, J., Macrocytic anemia in experimental cirrhosis. Proc. Staff Meet., Mayo Clinic, 1935, 10, 429.

7. Jacobson, B. M., On a pernicious anemia-like state in the guinea pig. J. Clin. Invest. (Proc.), 1934, 13, 714.

8. Jacobson, B. M., The response of the normal guinea pig to the administration of liver extracts. Science, 1934, 80, 211.

9. Jacobson, B. M., The response of the guinea pig's reticulocytes to substances effective in pernicious anemia; a biologic assay of the therapeutic potency of liver extracts. J. Clin. Invest., 1935, 14, 665.

10. Jacobson, B. M., The assay on guinea pigs of the hematopoietic activity of human livers, normal and pernicious anemia. J. Clin. Invest., 1935, 14, 679.

11. Jones, O. P., The reaction of normoblastic bone marrow to liver extract. J. Lab. and Clin. Med., 1936, 21, 335.

12. Landsberg, J. W., and Thompson, M. R., The guinea pig as a hematopoietic test animal. J. Am. Pharm. A., 1934, 23, 964.

13. Mermod, C., and Dock, W., A colloidal dye effective in treating pernicious anemia and evoking reticulocytosis in guinea pigs. Science, 1935, 82, 155.

14. Miller, D. K., and Rhoads, C. P., Absence of dietary anti-anemia substance in the diet causative of canine black tongue. Proc. Soc. Exper. Biol. and Med., 1934, 32, 419.

15. Miller, D. K., and Rhoads, C. P., The experimental production of loss of hematopoietic elements of the gastric secretion and of the liver in swine with achlorhydria and anemia. J. Clin. Invest., 1935, $14,153$.

16. Miller, D. K., and Rhoads, C. P., The reticulocyte response in guinea pigs following the oral administration of certain anti-anemic substances. New England J. Med., 1935, 213, 99.

17. Osgood, E. E., and Wilhelm, M. M., Reticulocytes. J. Lab. and Clin. Med., 1934, 19, 1129.

18. Pharmacopoeia of the United States of America, 11th Decennial Revision, Mack Printing Co., Easton, Pa., 1935, p. 484.

19. Rhoads, C. P., and Miller, D. K., The production in dogs of chronic black tongue with anemia. J. Exper. Med., 1933, 58, 585.

20. Subbarow, Y., Jacobson, B. M., and Fiske, C. H., The separation of the substances in liver which are reticulocytogenic in the guinea pig and which are therapeutically effective in experimental canine black tongue. New England J. Med., 1935, 212, 663. 